\title{
Fluid dynamics applications of IR imaging system
}

\author{
by L. DE LUCA, G. CARDONE and G.M. CARLOMAGNO
}

University of Naples, Faculty of Engineering, DETEC, P.le Tecchio 80, 80125 NAPLES, italy.

\begin{abstract}
The use of infrared thermography in several heat transfer and fluid dynamics problems is analysed and discussed. Different operating modes and their implementations are presented. Particular emphasis is given to the measurement of convective heat transfer coefficients. It is shown that, if the heat flux varies along the tested surface with relatively high spatial frequencies, the need arises of analysing the response of the heat flux sensors and of characterizing the spatial resolution of the IR imaging system.
\end{abstract}

\section{Nomenclature}

$\begin{array}{llll}D & \text { nozzle exit diameter } & T a w & \text { adiabatic wall temperature } \\ f & \text { restored output image } & T_{j} & \text { static temperature of jet } \\ G & \text { Fourier transform of input image } & T_{0} & \text { stagnation temperature } \\ h & \text { convective heat transfer coefficient } & T_{w} & \text { wall temperature } \\ H & \text { System Transfer Function } & T_{w i} & \text { initial foil temperature } \\ M & \text { Mach number } & V & \text { nozzle exit velocity } \\ N u & \text { Nusselt number } & x & \text { spatial coordinate } \\ p_{0} & \text { stagnation pressure } & y & \text { spatial coordinate } \\ Q & \text { total heat flux } & Z & \text { nozzle-to-plate distance } \\ Q c & \text { conductive heat flux } & \Delta T & \text { temperature difference } \\ Q r & \text { radiative heat flux } & \varepsilon & \text { surface emissivity coefficient } \\ r & \text { recovery factor } & \lambda & \text { thermal conductivity coefficient } \\ R & \text { radial coordinate } & \lambda f & \text { foil thermal conductivity coefficient } \\ R e & \text { Reynolds number } & \mu & \text { spatial frequency } \\ R e m & \text { unit free stream Reynolds number } & v & \text { spatial frequency } \\ s & \text { foil thickness } & \sigma & \text { Stefan-Boltzmann constant } \\ t & \text { time } & & \end{array}$

\section{Introduction}

In the last decade the good results attained by means of a widespread use of the Infrared Scanning Radiometer (IRSR) in the experimental study of convective heat transfer problems have proved IRSR itself to be an effective tool to overcome several limitations of the standard sensors, both from the measurement and the visualization points of view [1].

The exploitation of quantitative infrared (IR) thermography needs the solution of several problems mainly concerned with: an accurate characterization of the IR system performance and its calibration; the use of external additional optics and/or mirrors, e.g. to increase the spatial resolution; the choice of the most appropriate heat flux sensor and its characterization especially with regard to the lateral thermal conduction effects and to radiation losses; the determination of the body surface emissivity; the correct geometrical identification of the measured points; the design of the optical access window including the choice of the most appropriate IR material. An exhaustive treatment of these general aspects is made by CARLOMAGNO and DE LUCA [1] and BALAGEAS et al. [2]. 
This paper intends to discuss the use of the IR imaging system in various heat transfer and fluid dynamics problems. The applications herein discussed are selected to the aim of emphasizing some relevant questions that arise in the use of the IR technique.

\section{Measuring convective heat fluxes and visualizing flow fields}

When studying the problem at issue, a temperature difference is required between the flow and the tested body; it is therefore useful to make at first some remarks about the different experimental procedures to be followed depending on the flow Mach number $M$ range.

In the case of hyposonic flow regime $(M \ll 1)$, since the aerodynamic heating is not adequate to the sensitivity of $I R S R$, it is necessary to artificially produce a temperature difference between the model surface and the flow (active mode). This may be achieved either by varying the flow temperature or by heating the model in steady state or transient ways. A very convenient method to steadily heat models, having a cylindrical geometry of their surface, is the so-called heated-thin-foil technique. This consists of realizing (or coating) the model surface with a very thin metallic foil and heating it by Joule effect. By measuring the surface temperature, it is possible to compute the heat transfer coefficient from the foil to the flowing stream.

At high Mach numbers, because of the stream high kinetic energy content, the detection of the thermal image by IR thermography may be relatively simpler. In fact, the strong aerodynamic heating makes possible the use of the so-called passive mode. In general the model, which is initially at uniform ambient temperature, is suddenly exposed to the flowing stream. The thin-skin (e.g. CARLOMAGNO et al. [3]) or the thin-film (e.g. DE LUCA et al. [4]) heat flux sensors are generally used to obtain the convective heat transfer coefficients and/or to make diagnostics of the boundary layer. The possibility of evaluating the behaviour of the boundary layer over the surface of a body immersed in a flow by means of IR thermography derives from the close relation existing between skin friction and heat flux at the wall (Reynolds analogy).

When visualizing flow fields and/or measuring heat fluxes, the problem of restoring (in a general sense) the recorded thermal image (thermogram) may be very important both from the imaging and the measurement points of view. The thermogram, in fact, may be degraded both because of the limited spatial resolution of IRSR and due to the thermal conduction inside the heat flux sensor in the direction which is parallel to its surface. The first problem is addressed by ARCONADA et al. [5] and the more general one by DE LUCA and CARDONE [6]. Thermogram restoration can be performed by means of $F F T$ inverse filter algorithm:

$$
f(x, y)=j^{-1}\{G(\nu, \mu) / H(\nu, \mu)\}
$$

where $f(x, y)$ is the restored thermogram as a function of the spatial coordinates $x$ and $y, G$ is the Fourier transform of the recorded (degraded) image, and $H$ is referred to as the generalized System Transfer Function. Both $G$ and $H$ are functions of the spatial frequencies $v$ and $\mu$. Imaging and sampling as well as lateral thermal conduction effects may be accounted for by means of the Average Sampled System Modulation Transfer Function (ASSMTF) and of the Temperature Amplitude Transfer Function (TATF), respectively [6]. By assuming cascaded the above effects, the relationship $H=$ ASSMTF $\times$ TATF may be employed to solve equation (1).

\section{Natural convection over a vertical plate}

Some meaningful results concerned with the use of IRSR in natural convection studies are discussed. They deal with the transient natural convection over a heated vertical plate immersed in quiescent air which is subjected to a boundary condition of a step varying uniform heat flux. The heated-thin-foil technique is applied. Following CARDONE and CARLOMAGNO 
[7], the experimental set-up consists of a vertical thin stainless steel foil $(245 \mathrm{~mm}$ high, $960 \mathrm{~mm}$ wide, $40 \mu \mathrm{m}$ thick) electrically heated. IR camera records the thermal image of the plate at a distance of about $1 \mathrm{~m}$ through a lens of $20^{\circ}$ which gives a spatial resolution of about 0.5 pixels $/ \mathrm{mm}$.

The measured total emissivity of the foil back surface is relatively low $(\approx 0.15$, typical of polished stainless steel) but, since the one of the viewed surface is very high $(\approx 0.95$; in fact for measuring purposes this surface is coated with a thin layer of black paint), the radiation heat flux is about one third of the Joule heating, i.e. of the same order of magnitude of the convective one. Therefore the former one (from both surfaces) must be subtracted from the input electric power flux in order to evaluate the latter one. The radiation flux is computed by means of the same thermographic technique which proves to be very accurate in this case.

Tests are carried out for several values of the heat flux step $Q$ mostly starting from zero Joule heating. The transient natural convection heat transfer from a suddenly heated wall depends on the value of $Q$, on the wall thermal capacity and on the fluid properties. The natural convection regime, however, is always preceded by a regime of thermal conduction alone, whose duration increases as $Q$ decreases [7].

The plot of figure 1 reports the time trend of the temperature difference $\Delta T=T_{w}(t)-T_{w i}$ measured on the foil centerline at two vertical locations (from the foil leading edge), $y=61 \mathrm{~mm}$ and $169 \mathrm{~mm}$ for the power input step $0-130 \mathrm{~W} / \mathrm{m}^{2}$. Tw is the local wall temperature measured at time $t ; T w i$ is the initial foil temperature that, in the present case, coincides with the ambient one. Such trends are compared with the analytical prediction obtained by GOLDSTEIN and BRIGGS [8] for a pure conduction regime and by CARDONE and CARLOMAGNO [7] which include the effects of the radiative heat exchange. As shown by figure 1 , the presence of the radiative term (that is of the same order of magnitude of the conductive one) protracts of one order of magnitude the duration of the pure conduction regime; this latter appears longer at the highest vertical location. During the period in which the conductive mode alone is present, data well fit the analytical prediction of [7].

In the present natural convection studies, apart from the ones due to the strong radiation flux, no corrections were practically needed to take into account either the spatial resolution of the IR system or the effects of the lateral thermal conduction within the foil.

\section{Heat transfer from a plate to impinging jets}

Also the convective heat transfer to impinging jets is studied by means of the heated-thin-foil technique. CARLOMAGNO and DE LUCA [9] describe in detail the experimental apparatus and procedure. Being the Biot number (based on the foil thickness $s$ ) very small as compared to unity, the surface temperature distribution can be measured by viewing with the IR camera the rear face (opposite to the one where jets are impinging) of the foil. In order to increase the surface emissivity of the foil, this latter is covered by a thin film of black paint. Adiabatic wall temperature is assumed to coincide with the temperature exhibited by the foil when Joule heating is suppressed and it is measured by the very same thermographic technique.

Tests herein reported are performed for the case of a single jet. Truncated cone shaped nozzles, with rounded inlet sections and different nozzle exit diameters $D$ are generally used. The dimensionless nozzle-to-plate distance $Z / D$ and the Reynolds number $R e$ (based on $D$ and nozzle exit velocity $V$ are varied during the tests. Stagnation temperature of the jets is kept equal to ambient temperature by means of a heat exchanger.

The relief map of the recovery factor distribution $r=(T a w-T j) /(T o-T j)$ is reported in figure 2 for $R e=115,000 ; M=0.52 ; D=10 \mathrm{~mm} ; Z / D=8$. Taw is the adiabatic wall temperature and $T j$ and $T o$ are respectively the static and the total temperatures of the jet at nozzle exit. The recovery factor presents a local minimum in the annular region having a radius $R / D \cong 1$. This minimum is attributed to a vortex ring in the shear layer surrounding the jet and, as shown in figure 3 , it slightly moves outward as the distance $Z / D$ decreases. Data of figure 3 refer to the 
case of $D=5 \mathrm{~mm}, R e=65,000$ and $M=0.59$; they show, for different $Z / D$ values, the r radial profile which is symmetric with respect to the jet axis since it is obtained by performing averages in the azimuthal direction.

Data relative to the performed experimental studies on jets do not generally need imaging restoration. Furthermore, because of the small temperature differences that are encountered in the measurements of the adiabatic wall temperature, these latter do not practically need any correction for radiation heat exchange and for radial thermal conduction. The evaluation of the convective heat transfer coefficient $h$, instead, must be done by taking into account both radiation and conduction corrections:

$$
h=\left\{Q-\left[-\lambda f s\left(\frac{\partial^{2} T \mathrm{w}}{\partial R^{2}}+\frac{1}{R} \frac{\partial T_{\mathrm{w}}}{\partial R}\right)+\varepsilon \sigma\left(T_{\mathrm{w}}{ }^{4}-T_{0}{ }^{4}\right)\right]\right\} /\left(T_{\mathrm{w}}-T_{\mathrm{aw}}\right)
$$

where $\lambda \phi$ is the foil thermal conductivity, $\sigma$ the Stefan-Boltzmann constant, $s$ the foil thickness, $R$ the radial coordinate, $\varepsilon$ the surface emissivity.

The Nusselt number, defined as $N u=h D / \lambda$ where $\lambda$ is the air thermal conductivity coefficient evaluated at film temperature, gives the convective heat transfer coefficient in a dimensionless form. In figure 4 the radial distribution of Nusselt number is shown for $D=10 \mathrm{~mm}, Z / D=2, M=$ 0.13 and $R e=28,000$. Nu profiles are obtained by averaging data over circular rings. For such a short nozzle-to-plate distance there is an inner peak which occurs at $R / D=0.5$; the outer peak, located at $R / D \approx 2.5$, has the form of an annular hump. Also the minimum in between these two peaks can be attributed to the vortex ring in the shear layer surrounding the jet. The four profiles presented in figure 4 refer: to the coarse data $(Q)$, to those corrected for radiation alone $(Q-Q r)$ or conduction alone $(Q-Q c)$ and to those including both corrections $(Q-Q r-Q c)$. Differences between corrected and uncorrected data are small but not negligible.

\section{Görtler vortices in hypersonic flow}

Within the european community space program HERMES to develop the first european Space Shuttle, a series of experimental tests have been performed in a hypersonic wind tunnel (CEAT, Poitiers) on delta wing with ramp models. The operating fluid of tunnel is dry air which has typically a stagnation temperature $T o=800 \mathrm{~K}$ and a stagnation pressure $4.5 \mathrm{MPa}<p o<$ $10 \mathrm{MPa}$. Unit free stream Reynolds number Rem is changed mainly by varying the stagnation pressure and, in present tests, ranges from $.89 \times 107 / \mathrm{m}$ to $2.1 \times 10^{7} / \mathrm{m}$. The nominal free stream Mach number is equal to 8.15.

Particular attention is paid to visualize the presence of a transversal instability of the boundary layer over the ramp downstream of the delta wing, that leads to the formation of counter-rotating vortices in the reattaching flow, referred to as Görtler vortices [6]. The model, made of solid NORCOAT 4000 to measure heat fluxes by means of the thin-film technique, has a swept angle of $70^{\circ}$, a ramp angle of $15^{\circ}$ and a bottom wedge angle of $11^{\circ}$. At the beginning of each test run the model (which is initially in a remote position at room temperature) is vertically injected into the stream. A window, made of either germanium or zinc-selenide, AR coated is mounted on one side of the wind tunnel test section in order to provide an IR optical access to the tested model. A time sequence of thermograms (average time step is $0.24 \mathrm{~s}$ ) is generally recorded during the test runs. Injection time is about 0.15 s and testing duration of a few seconds.

The spanwise periodic variation of the convective heat transfer coefficient produces a variation of the model surface temperature which allows the detection of the vortices by means of the IR technique. Thermograms may also be used to visualize the overall surface flow. It is stressed that, owing to the relatively high spatial frequencies contained in the recorded thermal images, a thermogram restoration is strongly needed in this case. The recorded image, in fact, 
is degraded both because of the limited spatial resolution of the IR imaging system and due to the tangential thermal conduction inside the thin-film heat flux sensor.

The thermogram of figure A shows the restored temperature map relative to a square small frame $\left(21 \times 21 \mathrm{~mm}^{2}\right)$ viewed on the ramp downstream of the delta wing model. Testing conditions are: $M=8.15, R e=1.6 \times 10 \mathrm{E} 6(R e m=1.3 \times 10 \mathrm{E} 7 / \mathrm{m})$, zero angle of attack. Since the flow comes from the bottom of the thermogram, the presence of the spanwise vortices is made evident by the horizontal periodic variation of the temperature. The average wavelength of the vortices results to be about $2 \mathrm{~mm}$. The amount of the corrections due to the image restoration is shown in figure 5 where the Stanton number spanwise profile over the ramp (in particular along the line drawn on the thermogram of figure $A$ ) is reported. The dotted line represents the coarse data, the dashed line the ones restored from imaging and sampling degradation effects, the continuous line the overall restoration which takes into account also the lateral conduction degradation. As may be seen, in this specific case thermogram restoration is very important, particulary that involving imaging.

\section{Conclusions}

The capability of the $\mathbb{R}$ thermography to measure convective heat fluxes and to visualize flow fields has been analysed. The different experimental procedures to be followed, according to if the flow Mach number is relatively low or not, have been addressed. The needs of restoring the recorded thermal images (i.e., of removing the degradation effects), as well as of taking into account radiation losses of the sensor, have been also evidenced.

Applications to natural and forced convection problems have been reported. In the first one (study of free convection on a vertical plate in a transient state) it has been found that the presence of the radiative flux (which is also computed on the basis of IR measurement) determines a pure conduction heat transfer regime much longer than that for the case where this flux is negligible. The results concerning the application of IRSR to forced convection studies dealt with the mapping and the measurement of the cooling of a plate by means of impinging jets, as well as the aerodynamic heating in hypersonic flow. In particular, the detection of Görtler vortices in the reattaching flow region on a delta wing/ramp configuration furnished the opportunity of successfully applying a restoration technique which included imaging and measurement effects.

\section{REFERENCES}

[1] CARLOMAGNO (G.M.) and DE LUCA (L.). - Infrared thermography in heat transfer, in Handbook of Flow Visualization (Ed. Yang W.J.), Ch. 32, Hemisphere, 1989, p. 531-533.

[2] BALAGEAS (D.L.), BOSCHER (D.M.), DEOM (A.A). et FOURNIER (J.). - Application de la thermographie infrarouge passive et stimulée à la mesure des flux thermiques en soufflerie, La Recherche Aérospatiale, 1991-4, 1991, p. 51-72.

[3] CARLOMAGNO (G.M.), DE LUCA (L.) and ALZIARY DE ROQUEFORT (T.). - Mapping and measurement of aerodynamic heating and surface flow visualization by means of IR thermography, in Multiphase Flow and Heat Transfer (Eds. Chen, X.J. et al.), Vol. 2, Hemisphere, 1991, p. 1316-1324.

[4] DE LUCA (L.), CARDONE (G.), CARLOMAGNO (G.M.), AYMER DE LA CHEVALERIE (D.) and ALZIARY DE ROQUEFORT (T.). - Flow visualization and heat transfer measurement in hypersonic wind tunnel, Experimental Heat Transfer, Vol. 5, 1992, p. 65-79.

[5] ARCONADA (A.), ARGIRIOU (A.), PAPINI (F.) et PASQUETTI (R.). - La mesure en thermographie infrarouge: calibration et traitement du signal, J. Modern Optics, Vol. 34, 1987, p. 127-1335.

[6] DE LUCA (L.) and CARDONE (G.). - Experimental analysis of Görtler vortices in hypersonic wedge flow, in Thermosense XIV (Ed. J.K. Eklund), SPIE Vol. 1628, 1992, p. 21-281. 
[7] CARDONE (G.) and CARLOMAGNO (G.M.). - Convenzione naturale su lastra piana nei regimi stazionario ed instazionario, Atti IX Congresso Nazionale UIT, Pisa, 1991, p. 397-410.

[8] GOLDSTEIN (R.J.) and BRIGGS (D.G.). - Transient free convection about vertical plates and cylinders, Trans. ASME C, J. Heat Transfer, Vol. 86, 1964, p. 490-500.

[9] CARLOMAGNO (G.M.) and DE LUCA (L.). - Heat transfer to impinging jets Measured by infrared thermography, Proc. COBEM 89 (Eds. Hirata, M.H. et al.), Rio de Janeiro, 1989, p. 209-212. 
http://dx.doi.org/10.21611/qirt.1992.014

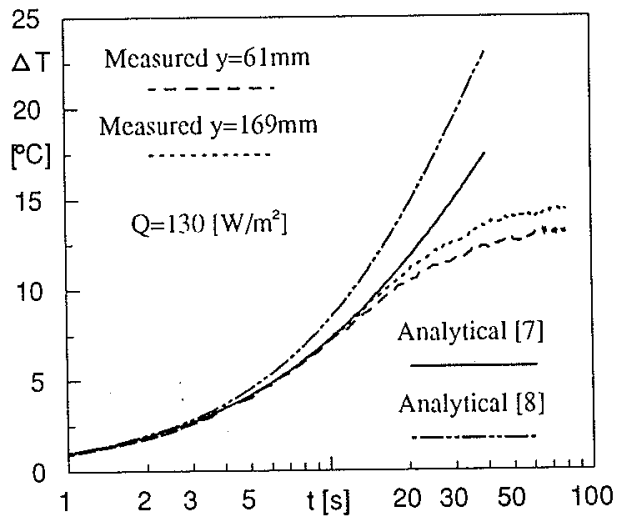

Fig. 1. - Temperature difference $\Delta T$ measured on the foil centerline

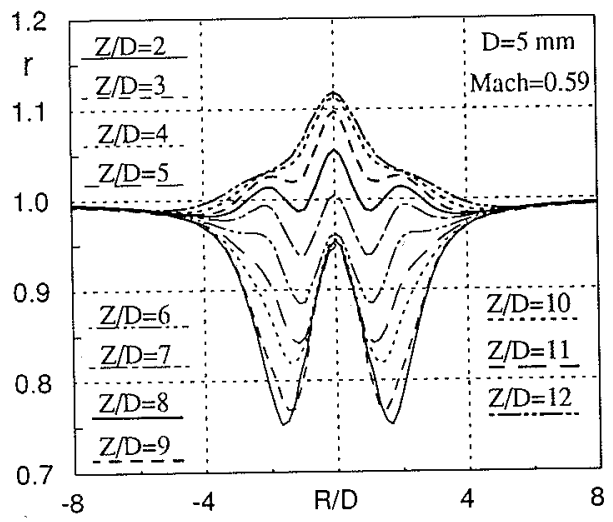

Fig. 3. - Recovery factor radial profiles

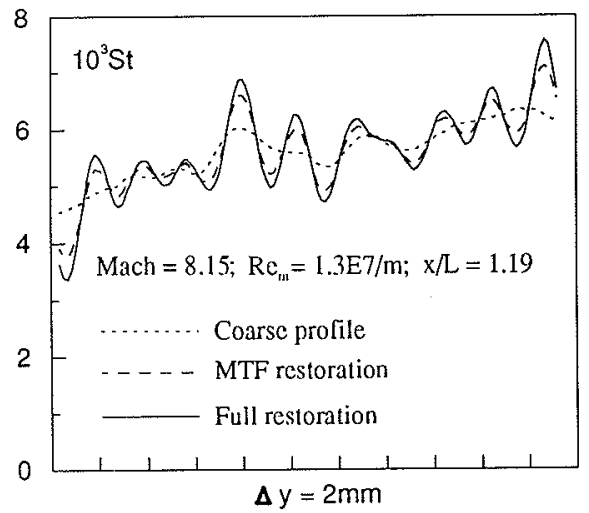

Fig. 5. - Stanton number spanwise profiles over the delta wing ramp

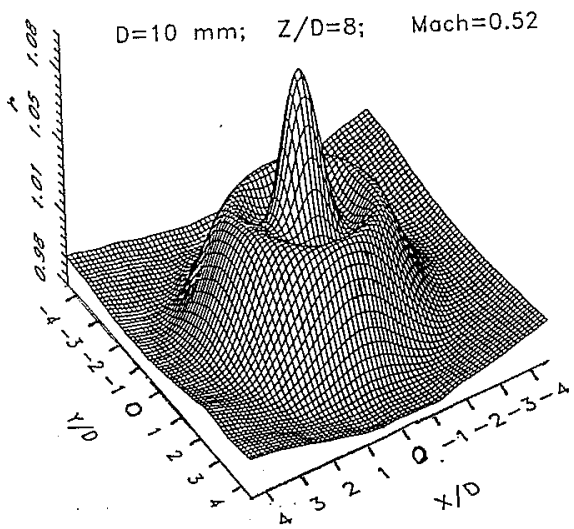

Fig. 2. - Relief map of the recovery factor

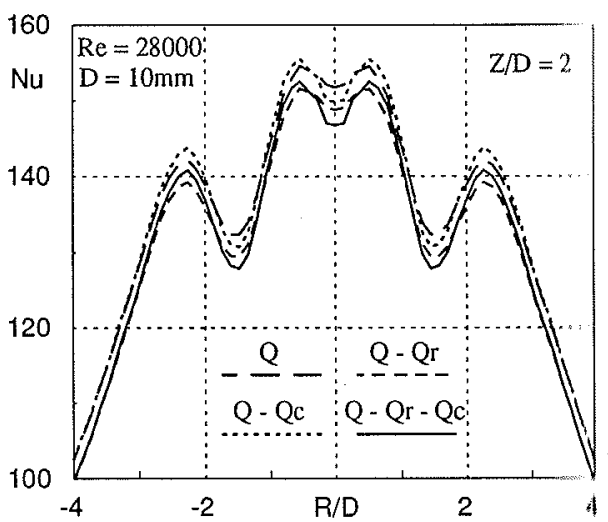

Fig. 4. - Nusselt number radial profilet 